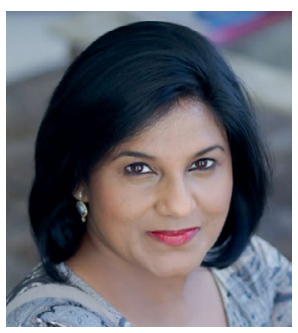

\title{
Vaccine inequity is unethical
}

\author{
Ethical principles dictate that limited, life-saving resources should be allocated fairly. \\ Keymanthri Moodley affirms that achieving global distributive justice is one of the greatest \\ challenges of the COVID-19 pandemic, and current distribution strategies are ethically \\ indefensible.
}

S carcity and resource constraints in healthcare are synonymous with life in Africa. Rationing and prioritization are obvious consequences of such scarcity. Ethically, such limited resources ought to be allocated fairly. Distributive justice was born out of such contexts: the fair distribution of dialysis machines, ventilators or critical care beds - scenarios that are all too familiar in Africa. At a domestic level, allocation frameworks are usually implemented by giving priority to high-risk groups who are most likely to benefit.

The COVID-19 pandemic, however, magnified the disproportion between supply and demand in healthcare at a global scale. Early in 2020, global desperation emerged for COVID-19 testing kits, personal protective equipment, intensive care beds and ventilators, all of which were preferentially supplied to countries with asymmetric purchasing power. COVID-19 vaccines soon became a highly sought-after commodity - a new global common interest.

Although COVID-19 vaccines are technically not regarded as a global public good using the criteria of non-rivalry and non-excludability, the production and deployment of preventative and therapeutic interventions during a pandemic is a matter of global public health ethics. Here the principles of limited autonomy, efficiency and social justice are central. Vaccine nationalism is rendered ethically indefensible on the basis of solidarity, reciprocity and interdependence. Yet, throughout the pandemic, self-interest has dominated the vaccine-distribution landscape.

Over the past two years, the disproportionate vaccine demand was exacerbated by global geopolitical forces that placed Africa last in line in the procurement of medical supplies. Lapses in global governance led to asymmetrical vaccine distribution, with high-income countries accessing and hoarding disproportionate quantities of limited vaccine supplies - a tragedy of the commons. Vaccine nationalism, despite being antithetical to the principles of distributive justice and solidarity, has flourished. Consequently, less than half the countries in Africa today have around 10\% of their adult population fully vaccinated. An even worse picture is emerging with respect to the asymmetry in allocation of third and fourth 'booster' doses.

Clearly, distributive justice, well-known in resource-poor domestic settings, has not been extrapolated to a global pandemic context.

Inequalities between high-income and low-and-middle-income countries transcend pre-COVID-19 disparities and vaccine access, and extend to a lack of reciprocity. Although the Omicron genomic data were deposited in the Global Initiative on Sharing Avian Influenza Data (GISAID) by scientists from South Africa for sharing with the global community, benefit-sharing agreements regarding boosters were not secured. Likewise, despite South Africa contributing important clinical trial data on vaccines to the global pandemic effort, the country was unable to secure fair pricing agreements for some vaccines - violating the fundamental principles of post-trial access and benefit sharing. These scenarios erode trust that is desperately needed to encourage vaccine uptake. As a result, even in African countries with reasonably good vaccine supplies, vaccine wastage and vaccine expiry are becoming sad realities owing to vaccine hesitancy and a small anti-vaccination movement. Vaccine wastage is compounded by appeals to solidarity with a resultant dumping of vaccine supplies on low-and-middle-income countries, often with extremely short expiry dates. These efforts plaster over deeper disparities in health across the globe, and do not follow from ethical considerations. With largely unvaccinated populations, Africa remains vulnerable to the risk of tourists bringing new variants to the continent despite their vaccination status.

What ought to have been an ethical response to global vaccine distribution? First, central custodianship ought to have rested with a powerful global health-governance body (such as the World Health Organization (WHO)) to ensure fair distribution of a global common resource, on the basis of risk, to all frontline healthcare and essential workers, globally. This ought to have been followed by the allocation of vaccines to individuals at risk of serious disease and death, globally. Although the WHO started a great pooled procurement initiative in the form of its 'COVID-19 Global Vaccine Access Facility’ (COVAX) to support global vaccine access, it was not sufficient to meet the global need in full and in time. High-income countries secured bilateral deals with vaccine manufacturers and proceeded with vaccine rollout, derailing vaccine delivery to COVAX.

Second, fostering self-reliance in Africa is critical to ramping up vaccine production. Temporary waivers on some intellectual property rights on COVID-19 vaccines ought to have been implemented to allow local manufacturers to produce vaccines. The application for waivers by South Africa and India has been blocked repeatedly by high-income countries that are essentially preventing universal access to vaccines. Failure to share vaccines equitably will lead to a vicious cycle with perpetuation of the pandemic, deteriorating global health and the undermining of our global economic recovery. Under such circumstances, our interdependence crumbles, destroying any hope of a sustainable healthy future for all.

Omicron has shown us how multilateralism and global social justice dissolved during the COVID-19 pandemic, and it has provided the evidence for why vaccine nationalism does not work, making this approach ethically indefensible. Sadly, what we are left with is a scenario of 'too 
little too late' for this global crisis, but perhaps - after more than five million deaths globally - this will serve as a costly dress rehearsal for the next public health emergency.
Keymanthri Moodley凶

Centre for Medical Ethics and Law, Department of Medicine, Faculty of Medicine and Health Sciences, Stellenbosch University, Stellenbosch, South Africa.

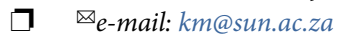

Published online: 31 January 2022

https://doi.org/10.1038/s41562-022-01295-w

Competing interests

The author declares no competing interests. 